\title{
Má percepção da limitação aos fluxos aéreos em pacientes com asma moderada a grave*
}

\author{
Adelmir Souza-Machado ${ }^{1}$, Manuela N. Cavalcanti ${ }^{2}$, Álvaro A. Cruz $^{3}$
}

\begin{abstract}
Introdução: Este estudo avaliou a percepção da obstrução das vias aéreas em pacientes ambulatoriais com asma moderada a grave e a capacidade da ausculta torácica em identificar a limitação aos fluxos aéreos. Métodos: Trinta e três pacientes foram avaliados em sete visitas semanais usando escores de sintomas por meio de escala visual analógica de sintomas (EVAS, 0-100mm), índice clínico de hiperreatividade brônquica (1-10), a classificação clínica de gravidade da asma (GINA, 1-4) e um escore de ausculta torácica (EAT, 0-5), espirometria e pico de fluxo expiratório (PFE), que foram correlacionados por meio do coeficiente de Spearman. Os pacientes foram classificados como percebedores $(-1 \leq r<0) e$ não percebedores $(0 \leq r \leq 1)$ através das correlações entre a EVAS para dispnéia e o $\mathrm{VEF}_{1}$. A correlação entre a ausculta e a obstrução brônquica foi considerada acurada quando um $r \leq-0,5$ (EAT vs. VEF ${ }_{1}$ ) era observado. Resultados: Dezessete asmáticos $(51,5 \%)$ não perceberam acuradamente o grau de obstrução das vias aéreas (não-percebedores). Nenhuma característica clínica pôde distinguir os grupos. Apenas $39,4 \%$ das correlações individuais entre EAT e $\mathrm{VEF}_{1}$ indicaram discriminação acurada pela ausculta. Asma grave não foi associada com ausculta não-acurada ou com má percepção neste estudo. Conclusão: Uma proporção significativa desta amostra de asmáticos não percebeu acuradamente a obstrução das vias aéreas. Além disso, o exame torácico mostrou ser um marcador inadequado da limitação aos fluxos aéreos em asmáticos moderados a graves, estáveis e ambulatoriais.
\end{abstract}

(J Pneumol 2001;27(4):185-192)

\section{Poor perception of airflow limitation in patients with moderate to severe asthma}

Background: This study evaluated the perception of airway obstruction in moderate to severely asthmatic outpatients and the efficiency of chest auscultation in identifying airflow limitation.

Methods: 33 subjects were evaluated at seven weekly visits by using symptoms scores determined by visual analogue scales (VAS, 0-100 mm), a clinical index of bronchial hyperreactivity (1-10), the clinical classification of asthma severity (GINA, 1-4) and a thoracic auscultation score (TAS, 0-5), spirometry and peak expiratory flow (PEF), which were correlated by the coefficient of Spearman.

Patients were classified as perceivers $(-1 \leq \mathrm{r}<0)$ or nonperceivers $(0 \leq \mathrm{r} \leq 1)$ by correlations between VAS for dyspnoea and $F E V_{1}$. A correlation between auscultation and bronchial obstruction was considered accurate when a $\mathrm{r} \leq-0.5$ (TAS vs. $\left.F E V_{1}\right)$ was found. Results: Seventeen asthmatic patients (51.5\%) did not accurately perceive the degree of their airways obstruction (nonperceivers). No clinical characteristics distinguished the groups. Only 39.4\% of the individual correlations between TAS and FEV 1 indicated accurate discrimination by auscultation. Severe asthma was not associated with inaccurate auscultation nor with malperception in this study. Conclusions: A significant proportion of this sample of asthmatic patients did not accurately perceive their own airway obstruction. Moreover, chest examination was shown to be an unsuitable discriminator of airflow limitation in moderate to severe stable asthmatics in an outpatient clinic.

* Trabalho realizado no Setor de Pneumologia, Hospital Universitário Professor Edgard Santos, Faculdade de Medicina da Universidade Federal da Bahia, Salvador, Bahia, Brasil.

1. Mestre em Imumologia Clínica; Professor Assistente de Farmacologia.

2. Médica Residente de Pneumologia do Pavilhão Pereira Filho-UFRGS.

3. Professor Titular de Clínica Médica; Coordenador da Disciplina de Pneumologia; Pesquisador do Serviço de Imunologia.
Endereço para correspondência - Álvaro A. Cruz, Disciplina de Pneumologia, Hospital Universitário Professor Edgard Santos, Rua João das Botas, s/n, $1^{\circ}$ andar - 40110-160 - Salvador, BA. Tel. (71) 339-6285; Fax (71) 237-6679; E-mail: alvaro@compos.com.br

Recebido para publicação em $20 / 10 / 00$. Aprovado, após revisão, em 1/6/01. 
Descritores - Asma. Obstrução das vias respiratórias. Auscultação. Pneumopatias obstrutivas.

Key words - Asthma. Airway obstruction. Auscultation. Lung obstructive diseases.

\section{INTRODUÇÃO}

Alguns pacientes são incapazes de perceber a intensidade da obstrução brônquica, subestimando a gravidade da doença ${ }^{(1,2)}$. Esses pacientes apresentam poucos sintomas, a despeito da intensa obstrução. A ausculta torácica também não tem demonstrado ser um instrumento acurado para detectar a gravidade do distúrbio obstrutivo ${ }^{(3,4)}$.

A dissociação entre sintomas e o grau de obstrução das vias aéreas é variável entre asmáticos ${ }^{(5)}$. Má percepção da obstrução brônquica tem sido detectada em $15 \%$ a $84 \%$ dos pacientes em vários estudos ${ }^{(1,6)}$. McFadden et al..$^{(7)}$ estudaram pacientes em fase de recuperação de asma aguda e observaram que os sintomas desaparecem antes da normalização da função pulmonar.

A capacidade do médico em identificar adequadamente a intensidade da obstrução brônquica em pacientes asmáticos depende dos sinais e sintomas das crises $^{(4)}$. Habitualmente, a presença de sibilos é o marcador da limitação aos fluxos aéreos disponível na prática clínica. Entretanto, a ausência desses ruídos adventícios ou a ausculta de outros sons pulmonares em pacientes asmáticos não constituem parâmetros confiáveis para aferir a obstrução brônquica ${ }^{(7,8)}$. A menor percepção da intensidade dos sons pulmonares à ausculta, nos asmáticos, é atribuída à limitação dos fluxos ${ }^{(9,10)}$.

A avaliação inadequada por médicos e pacientes pode retardar a identificação da obstrução brônquica, concorrendo para tratamento inadequado e mortes inesperadas dos pacientes.

O presente estudo destina-se a avaliar a percepção do grau da obstrução brônquica em pacientes ambulatoriais e estáveis, com asma moderada a grave, e a capacidade da ausculta torácica em identificar o grau da limitação aos fluxos aéreos nesses pacientes.

\section{CAsuística, MATERIAL E MÉTOdos}

Estudo prospectivo e descritivo que envolveu 36 asmáticos, ambulatoriais e clinicamente estáveis, acompanhados no ambulatório de asma do Hospital Universitário Prof. Edgard Santos, avaliados em sete visitas por seis semanas e tratados com esteróides tópicos (triancinolona $800 \mu \mathrm{g} / \mathrm{dia}$ ou beclometasona $1.000 \mu \mathrm{g} / \mathrm{dia}$ ) e beta-agonista inalado (salbutamol $400 / \mu \mathrm{g}$ ) de resgate. Foram selecionados asmáticos moderados a graves, diagnosticados havia pelo menos um ano(11). Todos os pacientes tinham $\mathrm{VEF}_{1}$ entre $40 \%$ e $80 \%$ do valor normal do previsto e resposta ao broncodilatador $>10 \%$. Não foram incluí-
Siglas e abreviaturas utilizadas neste trabalho

EAT - Escore de ausculta torácica

EVAS - Escala visual analógica de sintomas

ICHB - Índice clínico de hiper-reatividade brônquica

PFE - Pico de fluxo expiratório

CVF - Capacidade vital forçada

$\mathrm{VEF}_{1}$ - Volume expiratório forçado no $1^{\circ}$ segundo

$\mathrm{VEF}_{1} / \mathrm{CVF}$ - Razão entre o volume expiratório forçado no $1^{\circ} \mathrm{se}$ gundo e a capacidade vital forçada

$\mathrm{FEF}_{25-75 \%}$ - Fluxo expiratório entre os percentis 25 e 75

dos asmáticos em uso de esteróides orais ou inalatórios, agentes imunossupressores ou imunoterapia nos 30 dias que antecederam o estudo ou aqueles incapazes de seguir as recomendações do protocolo. Este estudo foi aprovado pelo Comitê de Ética Médica do Hospital Universitário Prof. Edgard Santos e consentimento pós-informação foi assinado por todos os participantes.

Os pacientes foram avaliados semanalmente por dois observadores distintos e independentes. O observador 1 (médico assistente) coletou as anamneses e realizou os exames físicos, determinando a classificação da gravidade da asma de acordo com os critérios do GINA ${ }^{(11)}$. A ausculta torácica foi registrada como um escore de ausculta torácica (EAT) ${ }^{(12)}$. Em adição, foi aplicado um questionário para obtenção do índice clínico de hiper-reatividade brônquica $(\mathrm{ICHB})^{(13)}$; o observador 2 (estudante de medicina) aplicou a escala visual analógica de sintomas (EVAS)(14), obteve manobras espirométricas, revisou o pico de fluxo expiratório (PFE) e orientou os pacientes sobre como anotar os valores encontrados no cartão diário. Todos os procedimentos de avaliação eram repetidos todas as semanas até o final do estudo.

O EAT era obtido durante a ausculta em dois momentos distintos (basal e 15 minutos após broncodilatador) e obedeceu a uma escala de 0 a 5: $0=$ murmúrio vesicular (MV) normal, sem ruídos adventícios; 1 = MV diminuído, sem ruídos adventícios; $2=\mathrm{MV}$ diminuído, com sibilos à expiração forçada; 3 = MV diminuído, com sibilos leves e esparsos à expiração normal; 4 = sibilos intensos ou difusos; 5 = uso de musculatura acessória ou tiragem. Toda a área do tórax foi auscultada e o maior escore registrado para a análise.

$\mathrm{O}$ ICHB é um escore baseado em questionário aplicado aos pacientes antes do exame físico, para identificar desencadeantes de sintomas, na semana anterior à visita, entre dez agentes: poeira, mofo, odores fortes, fumaça, alterações climáticas, infecção de vias aéreas, alimentos, exercícios, emoções e uso de aerossol. Era atribuído valor 1 para cada item presente, com somatório máximo de dez e mínimo de zero.

A intensidade dos sintomas (tosse, dispnéia, opressão e chiado) durante as consultas era avaliada por meio de 
uma escala visual analógica de sintomas ${ }^{(14)}$. A escala utilizada era uma linha horizontal de $100 \mathrm{~mm}$ em que em um extremo representava a ausência de sintomas $(0 \mathrm{~mm})$ e o outro a presença máxima (100mm). Os intervalos marcados pelos pacientes eram medidos em milímetros e registrados para análise.

As espirometrias eram realizadas em todas as visitas com o espirômetro Microlab 3300 (Micro Direct Inc., UK) de acordo com a técnica recomendada pela ATS(15). As manobras eram repetidas 15 minutos após o uso de salbutamol inalado $(400 \mu \mathrm{g})$. Os dados obtidos foram expressos em termos absolutos ( $\mathrm{VEF}_{1} / \mathrm{CVF}$ ) e como percentagem do valor normal do previsto (CVF, $\mathrm{VEF}_{1}, \mathrm{FEF}_{25-75 \%}$ ). O PFE (Mini Wright AFS) era medido duas vezes ao dia, em triplicata, durante todo o protocolo e em cada visita (PFE extra).

\section{Análise estatística}

Para avaliar a percepção da obstrução das vias aéreas, parâmetros clínicos tais como escala analógica visual de sintomas $^{(14)}$, índice clínico de hiper-reatividade brônqui$\mathrm{ca}^{(13)}$ e a classificação clínica da gravidade da asma ${ }^{(11)}$ foram correlacionados com dados da função pulmonar (CVF, $\mathrm{VEF}_{1}, \mathrm{VEF}_{1} / \mathrm{CVF}, \mathrm{FEF}_{25-75 \%}$ e PFE) utilizando o teste de correlação de Spearman. Os pacientes foram classificados arbitrariamente como percebedores $(-1 \leq r<0)$ e não-percebedores $(0 \leq r \leq 1)$ através das correlações individuais entre EVAS (dispnéia) e VEF. Para a avaliação da capacidade da ausculta em identificar a gravidade da obstrução brônquica, foi observada a correlação do EAT com o $\mathrm{VEF}_{1}$ para cada paciente; foram considerados discriminação acurada coeficientes de correlação $\leq-0,5$.

Os testes não-paramétricos de Mann-Whitney e Wilcoxon, Wilcoxon matched pairs signed ranks e o teste de Friedman foram utilizados para comparar distribuições.
O teste do qui-quadrado foi escolhido para comparar proporções. Consideraram-se significância estatística os valores de $\mathrm{p} \leq 0,05$.

\section{RESULTADOS}

\section{Características clínicas}

Foram relacionados 36 pacientes, mas três foram excluídos (um por registro incorreto do PFE e dois por ter usado corticosteróides orais). Trinta e três pacientes completaram o protocolo ( $24 \mathrm{~F}$ e $9 \mathrm{M})$, com idades que variaram de 13 a 73 anos (média \pm erro padrão: 38,5 $\pm 2,9$ anos; Tabela 1). Nenhum dos pacientes fumava. As características clínicas foram sumarizadas na Tabela 1.

Dezessete asmáticos $(51,5 \%)$ foram classificados como não-percebedores e $16(48,5 \%)$ como percebedores $(\mathrm{p}=$ 0,82 ; Tabela 1) de acordo com os critérios preestabelecidos.

\section{Escores de sintomas e ICHB}

$\mathrm{Na}$ análise intergrupos, os escores de dispnéia, tosse, chiado e opressão no peito foram superiores nos percebedores quando comparados com os não-percebedores. Todas essas diferenças foram significantes (Tabela 2). No grupo de percebedores o escore de dispnéia foi superior ao dos demais escores de sintomas $(20,5 \mathrm{~mm})$, situação não observada no grupo de não-percebedores, em que o escore de tosse $(6,1 \mathrm{~mm})$ foi o mais elevado. O ICHB não diferiu entre os grupos (Tabela 2).

\section{Dissociação entre sintomas e limitação ao fluxo aéreo}

Os parâmetros espirométricos (média\% \pm erro padrão dos valores preditos) da amostra total $(\mathrm{n}=33$ ) foram: $\mathrm{CVF}$ $(80 \% \pm 3,3 \%), \mathrm{VEF}_{1}(60,6 \% \pm 3 \%), \mathrm{VEF}_{1} / \mathrm{CVF}(75,5 \% \pm$

TABELA 1

Características clínicas da amostra estudada

\begin{tabular}{|c|c|c|c|}
\hline Características & $\begin{array}{c}\text { Todos } \\
(n=33)\end{array}$ & $\begin{array}{l}\text { Percebedores } \\
\quad(n=16)\end{array}$ & $\begin{array}{l}\text { Não-percebedores } \\
\qquad(n=17)\end{array}$ \\
\hline Sexo & $9 \mathrm{M}, 24 \mathrm{~F}$ & $5 \mathrm{M}, 11 \mathrm{~F}$ & $4 \mathrm{M}, 13 \mathrm{~F}$ \\
\hline Idade (anos) $)^{\sharp}$ & $38,5 \pm 2,9$ & $43,9 \pm 4,0$ & $33,4 \pm 3,8$ \\
\hline \multicolumn{4}{|l|}{ Grupo racial (\%) } \\
\hline Negros & $22(66,7)$ & $12(75,0)$ & $10(58,8)$ \\
\hline Não-negros & $11(33,3)$ & $4(25,0)$ & $7(41,2)$ \\
\hline Asma familiar (\%) & $16(48,5)$ & $9(56,3)$ & $7(41,2)$ \\
\hline Atopia (\%) & $11(33,3)$ & $6(37,5)$ & $5(29,4)$ \\
\hline Duração da asma (anos) $)^{\sharp}$ & $11,2 \pm 0,3$ & $11,4 \pm 0,2$ & $11,1 \pm 0,5$ \\
\hline \multicolumn{4}{|l|}{ Gravidade da asma (n) } \\
\hline Moderado/grave & $15 / 18$ & $6 / 10$ & $9 / 8$ \\
\hline
\end{tabular}


TABELA 2

Avaliação global da escala visual analógica de sintomas* (EVAS) e o índice clínico de hiper-reatividade brônquica (ICHB)

\begin{tabular}{lrrrr}
\hline & $\begin{array}{c}\text { Todos } \\
(\mathbf{n}=\mathbf{3 3})\end{array}$ & $\begin{array}{c}\text { Percebedores } \\
(\mathbf{n}=\mathbf{1 6})\end{array}$ & $\begin{array}{c}\text { Não-percebedores } \\
(\mathbf{n}=\mathbf{1 7})\end{array}$ & $\mathbf{p}^{* *}$ \\
& & & & \\
\hline & & & \\
Tosse & $11,8 \pm 2,3$ & $17,9 \pm 3,8$ & $6,1 \pm 2,1$ & 0,017 \\
Chiado & $10,1 \pm 2,2$ & $16,6 \pm 3,6$ & $4,0 \pm 1,4$ & 0,042 \\
Opressão & $10,3 \pm 2,2$ & $16,5 \pm 3,5$ & $4,5 \pm 1,8$ & 0,003 \\
Dispnéia & $11,6 \pm 2,4$ & $20,5 \pm 3,6$ & $3,2 \pm 1,5$ & 0,0001 \\
ICHB & $3,2 \pm 0,3$ & $3,4 \pm 0,3$ & $3,0 \pm 0,4$ & 0,26 \\
\hline
\end{tabular}

$\S$ Média \pm erro padrão.

* Escore de sintomas da escala visual exibidos em milímetros ( 0 a 100mm).

** $\mathrm{p}=$ significância estatística entre percebedores e não-percebedores.
$1,8 \%)$ e $\mathrm{FEF}_{25-75 \%}(33,7 \% \pm 2,6 \%)$. Para o PFE foi o seguinte [média (variação)]: PFE matinal [51,2\% (23,6\% a $93,5 \%)]$, variação diária do PFE [14,7\% (4,3\% a 28,8\%)] e o PFE extra, no momento da consulta $[61,8 \%(34,9 \%$ a $102,6 \%)]$. A função pulmonar não diferiu entre os percebedores e não-percebedores. Algumas dessas medidas são exibidas na Figura 1.

No grupo de não-percebedores, foi observada surpreendente dissociação entre o valor do $\mathrm{VEF}_{1}$ e o escore de dispnéia, representada por correlação direta e fraca, sem significância estatística $(r=0,36 ; p=0,15)$. Em ambos os grupos, percebedores e não-percebedores, os valores percentuais do previsto de $\mathrm{VEF}_{1}$ e de $\mathrm{VEF}_{1} / \mathrm{CVF}$ mostraram-se reduzidos, mas estáveis, e apresentaram poucas
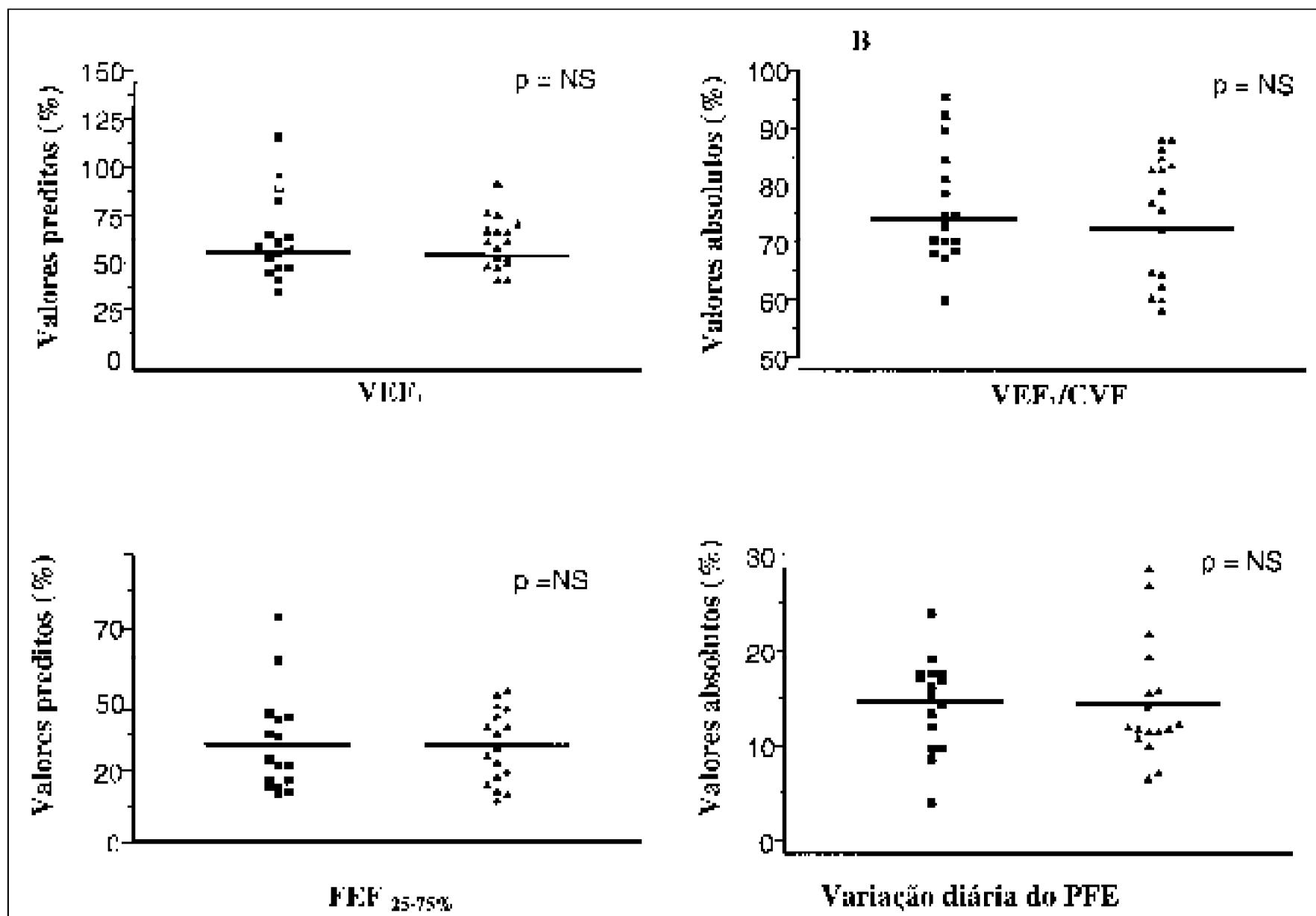

Percebedores

A Nāo-percebedores

NS = não significante. Testes de Mann-Whitney não apresentaram diferenças entre os grupos. Barras horizontais indicam a média.

Figura 1 - Função pulmonar dos pacientes de acordo com a percepção da obstrução brônquica 


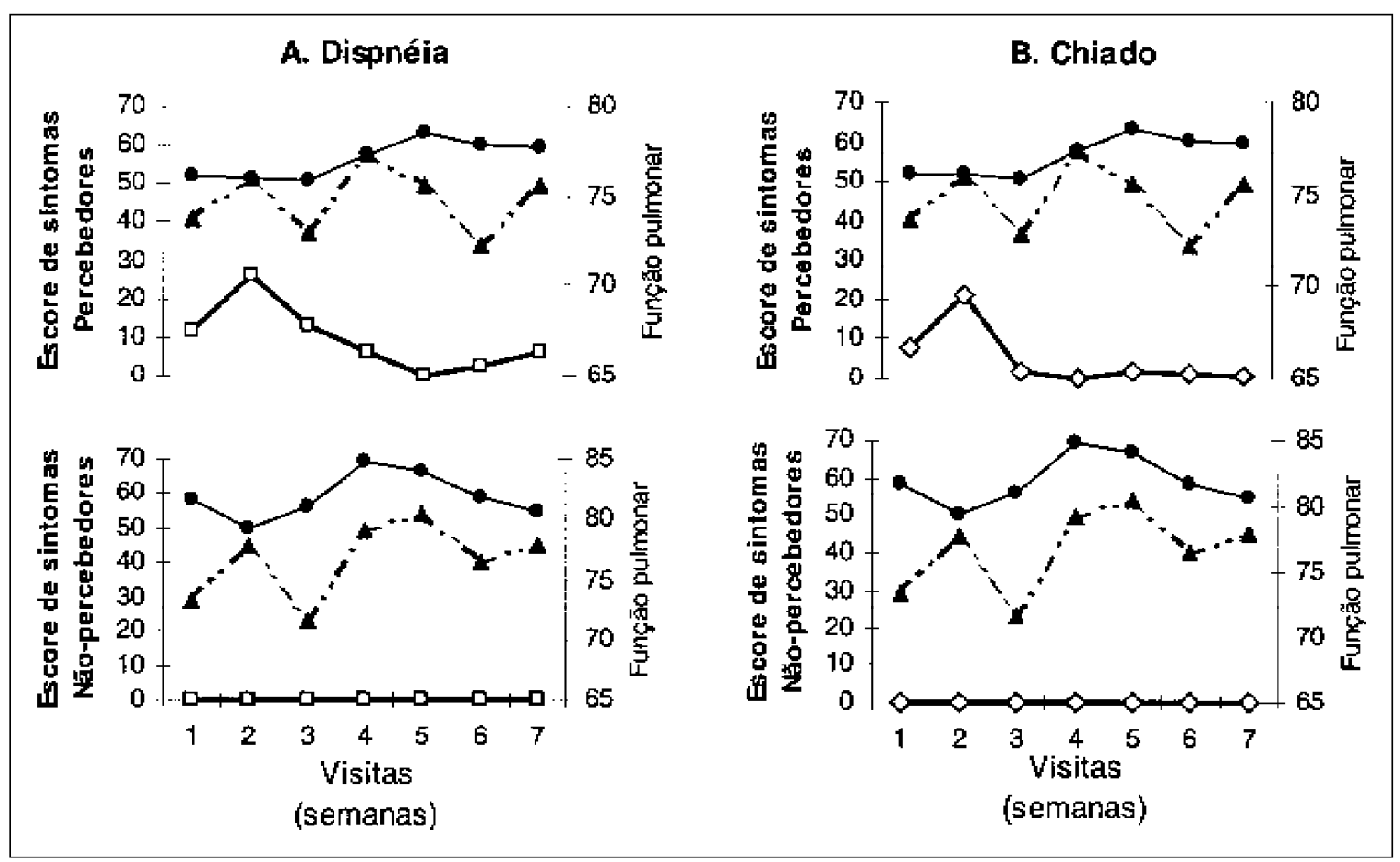

Figura 2 - Evolução dos sintomas comparados com a função pulmonar. Todos os dados são expressos como medianas. Os círculos representam o $V E F_{1} / C V F$; triângulos, $V E F_{1}$; os quadrados abertos, dispnéia, e os losangos abertos, o chiado no peito.

variações durante o estudo (Figuras 2A e 2B). Quando foi analisada a evolução dos sintomas, as medianas dos escores de sibilos e dispnéia foram zero em todas as visitas testadas durante o protocolo no grupo de não-percebedores (Figura 2B). Os sintomas dos percebedores (chiado e dispnéia) diminuíram progressivamente até o término do protocolo (Figura 2B).

\section{Dissociação entre a ausculta torácica e intensida- de da obstrução brônquica}

Foi observada ampla variação individual dos coeficientes de correlação; apenas 39,4\% das correlações indicaram discriminação acurada (EAT vs. $\mathrm{VEF}_{1}$; Figura 3). As freqüências de discriminação acurada para as outras correlações observadas foram: EAT vs. PFE matinal $(51,5 \%)$, EAT vs. PFE extra $(42,4 \%)$ e do EAT vs. $\operatorname{FEF}_{25-75 \%}(42,4 \%)$.

\section{Relação entre ausculta torácica e distúrbio obs- trutivo}

Os dados dos pacientes foram relacionados em dois grupos, de acordo com a capacidade da ausculta pulmonar em identificar a gravidade da obstrução brônquica: ausculta acurada $(r \leq-0,5)$ e ausculta não-acurada. As variáveis idade, tempo de duração da asma e escore de chiado no peito foram similares entre os grupos (dados não mostrados).

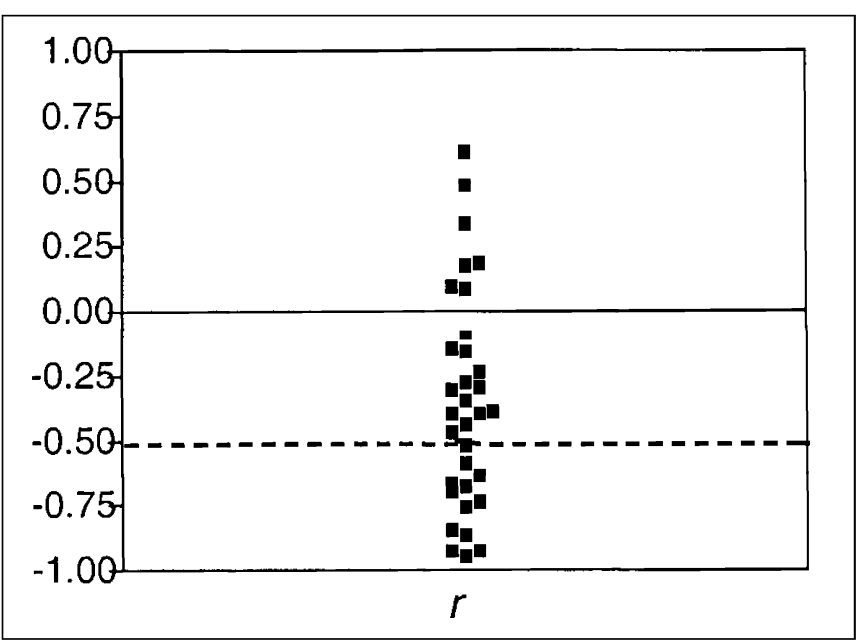

Figura 3 - Coeficientes de correlação (Spearman) entre o escore de ausculta torácica e o volume expiratório forçado no primeiro segundo. Correlações individuais para 33 pacientes asmáticos durante o estudo, no qual apenas $39,4 \%$ apresentaram $r \leq-0,5$.

As medidas espirométricas não diferiram estatisticamente quando comparados os grupos de ausculta não-acurada e acurada: [média\% (erro padrão); CVF: $82,5 \%(4,8)$ vs. $76,3 \%(2,9) ; \mathrm{VEF}_{1}: 65,3 \%(4,6)$ vs. $54,8 \%(2,3) ; \mathrm{VEF}_{1} /$ CVF: $77,3 \%(2,2)$ vs. $72,6 \%(3,0)$; resposta ao BD ao nível 


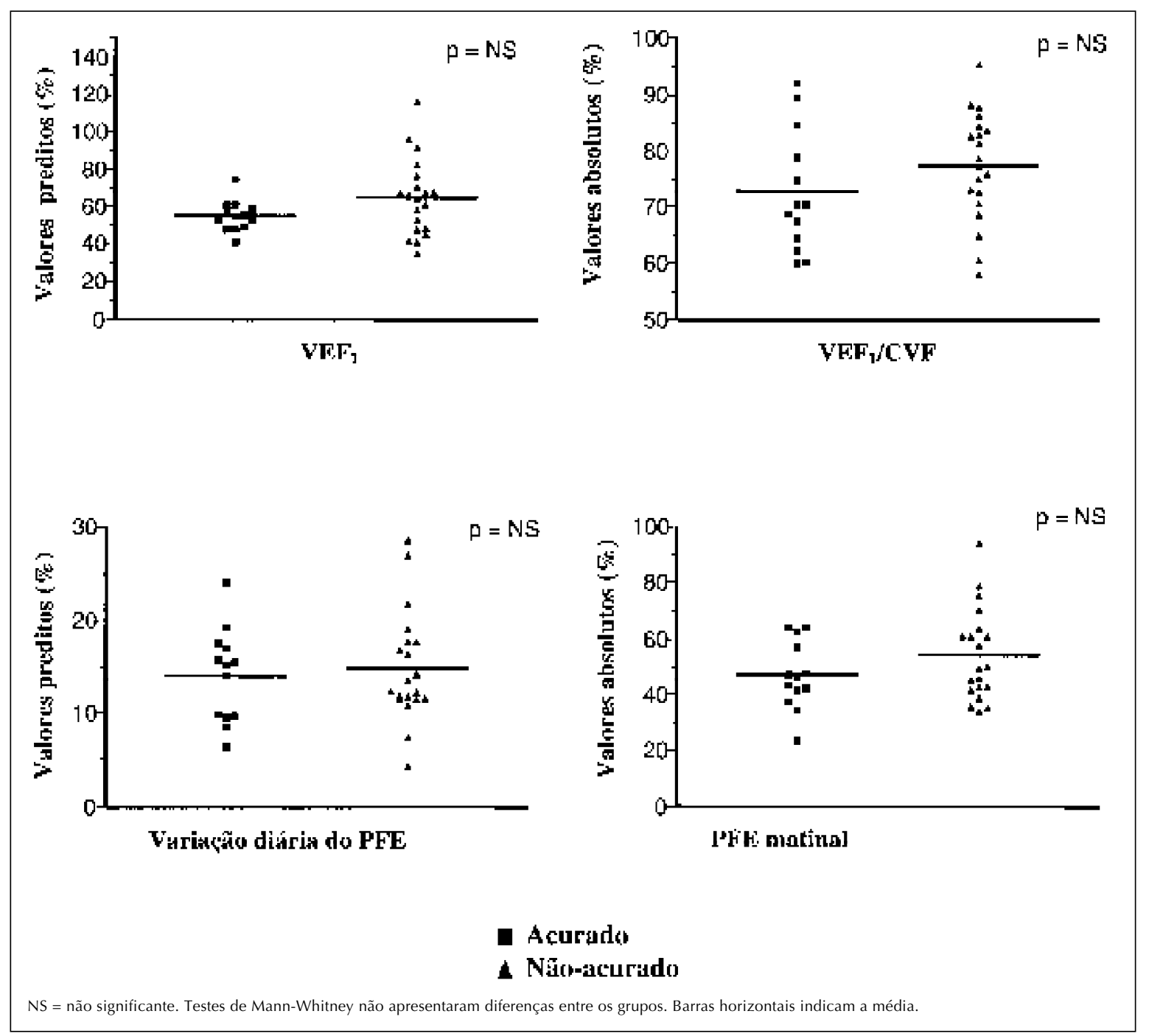

Figura 4 - Função pulmonar e acurácia da ausculta torácica

de $\mathrm{VEF}_{1}: 10,5 \%(1,2)$ vs. $11,4 \%(1,7)$ e $\mathrm{FEF}_{25-75 \%}: 37,1 \%$ $(3,9)$ vs. $28,3 \%(2,5)]$ Resultados similares foram observados para o PFE (Figura 4). A gravidade da asma também não se associou aos escores de ausculta torácica em 221 observações ao longo do acompanhamento. A ausculta não-acurada associou-se a asma grave em apenas $31,7 \%$ das ocasiões.

Observou-se correlação inversa e estatisticamente significante entre o EAT e o PFE geral $(r=-0,44, p=0,01$; Figura 5) embora o valor do coeficiente fosse inferior àquele considerado como discriminação acurada pelo método de ausculta $(r \leq-0,5)$.

\section{DISCUSSÃO}

Observamos que proporção significativa dos pacientes com asma moderada a grave, estáveis e ambulatoriais,

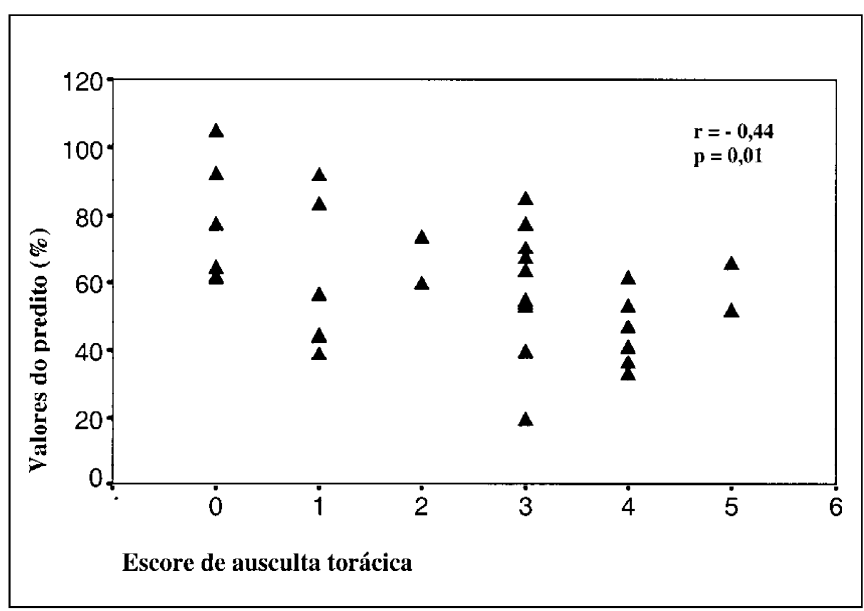

Figura 5 - Correlação entre o escore de ausculta torácica e pico de fluxo expiratório (PFE extra), no momento da consulta 
não percebeu acuradamente a gravidade da obstrução de suas vias aéreas. A maioria das correlações entre os escores de ausculta e o $\mathrm{VEF}_{1}$ situou-se em valores que indicaram discriminação inadequada da intensidade da limitação aos fluxos aéreos pelo exame físico.

Mais da metade dos pacientes não identificou acuradamente o grau de obstrução de suas vias aéreas e, dessa forma, foram classificados como não-percebedores. O teste de correlação de Spearman foi utilizado para determinar a percepção da obstrução brônquica, pelo pacientes, em um intervalo $(-1 \leq r<0)$ que permitisse identificar mínimas associações entre os sintomas relatados $e$ os parâmetros de função pulmonar.

Não foram identificadas características clínicas ou demográficas que distinguissem os grupos de percebedores e não-percebedores, em concordância com o observado por outros autores ${ }^{(1-2,17-19)}$. No presente estudo, não existiram diferenças entre as médias de idade, duração da doença, freqüência de atopia ou história familiar de asma. Todavia, alguns autores associaram má percepção da broncoconstrição a pacientes com idade elevada ${ }^{(17)}$ e com maior tempo de duração da doença ${ }^{(18)}$. Alguns fatores intrínsecos têm sido atribuídos a má percepção da limitação aos fluxos aéreos na asma, tais como os volumes pulmonares ${ }^{(19)}$, a sensibilidade dos quimiorreceptores ${ }^{(20)} e$ a intensidade da inflamação brônquica ${ }^{(16,20)}$, mas não foi a intenção deste trabalho explorar essa questão.

Nossa contribuição para o atual conhecimento neste assunto está relacionada aos seguintes aspectos: 1) a nossa amostra, asmáticos moderados a graves estáveis; 2) a combinação da avaliação da percepção do paciente e a discriminação da ausculta; e 3) a monitorização repetida do $\mathrm{VEF}_{1}$, da ausculta e da escala visual analógica de sintomas em pacientes ambulatoriais.

Existem estudos descrevendo associações entre a má percepção da obstrução brônquica, gravidade da doença ${ }^{(16,18)}$ e risco elevado de asma fatal(21). No presente estudo, foram observadas proporções iguais de percebedores e não-percebedores nos grupos classificados como asma moderada e grave, sugerindo que a gravidade da asma não está relacionada a má percepção. Além disso, observou-se que a reatividade das vias aéreas quando aferida pelo ICHB foi similar em ambos os grupos. As observações de Salome et al. ${ }^{(22)}$ e Laprise e Boulet ${ }^{(23)}$ mostraram que a hiper-reatividade brônquica não parece interferir na percepção da obstrução das vias aéreas em pacientes não-asmáticos.

A escala visual analógica de sintomas é um recurso utilizado para tornar menos subjetiva a mensuração de alguns sintomas relatados pelos pacientes, tais como dispnéia, dor e fadiga ${ }^{(14)}$. É um instrumento muito sensivel e de fácil aplicação. Os escores de sintomas (dispnéia, tosse, chiado e opressão no peito) foram superiores na aná- lise global em percebedores quando comparados com os não-percebedores. Estes resultados podem ser considerados previsiveis pela natureza do estudo. No decorrer do protocolo, dispnéia foi o sintoma com maiores escores durante o tratamento. Os percebedores obtiveram redução nas medianas dos escores de dispnéia, mas isso não foi observado em não-percebedores, que exibiram medianas de zero em todas as semanas testadas. Considerando-se que a função pulmonar foi similar (aproximadamente $60 \%$ dos valores normais do predito) em todos os asmáticos - percebedores e não-percebedores - $e$ os escores de sintomas decresceram no decorrer do estudo em percebedores, pode-se assumir que os sintomas de asma podem ser reduzidos pelo tratamento antes da melhora da função pulmonar, como observado previamente por outros autores ${ }^{(6-7)}$. Essas observações poderiam também resultar da obstrução persistente aos fluxos aéreos em pequenos brônquios periféricos que não produz sintomas em repouso, mas altera a função pulmonar.

\section{Dissociação entre ausculta e intensidade da limi- tação aos fluxos aéreos}

No presente estudo, apenas $39,4 \%$ dos pacientes apresentaram correlações que representaram discriminação acurada. Esses resultados evidenciaram que o método de ausculta não foi suficiente para identificar a gravidade da obstrução brônquica na maioria dos pacientes. A correlação do EAT versus PFE matinal exibiu o maior número de coeficientes considerados acurados $(\mathrm{r} \leq-0,5)$, mas ainda em uma freqüência de apenas $51 \%$.

A detecção de respiração ruidosa pode ser evidenciada freqüentemente quando a redução do $\mathrm{VEF}_{1}$ se situa entre 35 e $40 \%^{(24)}$. Em obstruções graves o colapso do brônquio pode reduzir as oscilações das paredes brônquicas e impedir a presença de sibilos ${ }^{(25)}$. Também as modificações estruturais dos brônquios provocadas pela inflamação e edema podem alterar a transmissão dos sons através do tórax e a percepção pelo método de ausculta. Contudo, no presente estudo, asma grave não se associou com a ausculta não-acurada.

Parâmetros objetivos de função pulmonar são importantes para avaliação da intensidade da obstrução brônquica em asmáticos. No presente estudo, a função pulmonar foi similar em ambos os grupos - ausculta acurada e não-acurada, o que sugere que diferentes fatores podem estar envolvidos na capacidade da ausculta em identificar a limitação dos fluxos aéreos. As variações poderiam ser devidas ao método utilizado, características dos sons pulmonares gerados, alterações morfológicas nos brônquios e a experiência do observador ${ }^{(4,8,24-26)}$. O desaparecimento dos sinais e sintomas precedeu a normalização da função pulmonar, na asma aguda, como demonstrado por McFadden et al. ${ }^{(7)}$. 
Algumas publicações têm chamado atenção para a relevância da má percepção da obstrução brônquica em asmáticos $^{(1-4,8-10,16-18)}$. Nossos resultados confirmam essas observações em pacientes com asma moderada a grave, ambulatoriais e estáveis, utilizando repetidamente as ferramentas clínicas mais acuradas disponíveis na prática diária: as escalas visuais analógicas de sintomas e a espirometria. Tais circunstâncias podem aumentar a probabilidade da validade externa de nossas observações.

Em resumo, observou-se que mais da metade dos pacientes ambulatoriais e estáveis, com asma moderada a grave, não perceberam o grau de obstrução de suas vias aéreas. Não encontramos características clínicas que os identificassem.

Em relação à capacidade do médico em determinar a gravidade da obstrução brônquica, observamos que a maioria das correlações entre os escores de ausculta e o $\mathrm{VEF}_{1}$ indicou discriminação inadequada por este método. Má percepção por pacientes e médicos pode atrasar a identificação da obstrução brônquica, retardar o tratamento adequado e contribuir para mortes inesperadas por asma. Portanto, mensurações objetivas da limitação aos fluxos aéreos são cruciais para a avaliação de pacientes com asma moderada a grave no local mais relevante, que é o cenário da visita ao consultório quando o médico tem que decidir sobre o tratamento.

\section{REFERÊNCIAS}

1. Rubinfeld AR, Pain MCF. Perception of asthma. Lancet 1976;24:882883.

2. Kendrick AH, Higgs CMB, Whitifield MJ, Laszlo G. Accuracy of perception of severity of asthma: patients treated in general practice. $\mathrm{Br}$ Med J 1993;307:422-424.

3. Shim CS, Williams MH. Relationship of wheezing to the severity of obstruction in asthma. Arch Intern Med 1983;143:890-892.

4. Baumann UA, Haerdi E, Keller R. Relations between clinical signs and lung function in bronchial asthma: how is acute bronchial obstruction reflected in dyspnoea and wheezing? Respiration 1986;50:294-300.

5. Bellofiore S, Ricciardolo FLM, Ciancio N, Sapienza MA, Patane A, Mistretta A, Di Maria GU. Changes in respiratory drive account for the magnitude of dyspnoea during bronchoconstriction in asthmatics. Eur Respir J 1996;9:1155-1159.

6. Atherton HA, White PT, Hewett G, Howells K. Relationship of daytime asthma symptom frequency to morning peak expiratory flow. Eur Respir J 1996;9:232-236.

7. McFadden Jr ER, Kiser R, Degroot WJ. Acute bronchial asthma. Relations between clinical and physiologic manifestations. N Engl J Med 1971;288:221-225.
8. Bohadana AB, Massin N, Teculescu D, Peslin R. Tracheal wheezes during methacholine airway challenge (Mac) in workers exposed to occupational hazards. Respir Med 1994;88:581-587.

9. Shim CS, Williams MH. Evaluation of the severity of asthma: patients versus physicians. Am J Med 1980;68:11-13.

10. Schreur HJ, Sterk PJ, Vanderschoot J, van Klink HC, van Vollenhoven E. Lung sound intensity in patients with emphysema and in normal subjects at normalized airflow. Thorax 1992;47:674-679.

11. NHLBI, National Heart, Lung and Blood Institute-World Health Organization. Pocket Guide for Asthma Management and Prevention. Maryland, 1995;23p.

12. Sestelo M. Escore de ausculta torácica na identificação do grau de obstrução das vias aéreas em asmáticos. Dissertação [Mestrado, Medicina Interna] Salvador: Faculdade de Medicina da Universidade Federal da Bahia, 1997;79p.

13. Araújo NN. Valor de um índice clínico de hiper-reatividade brônquica no acompanhamento de pacientes com asma. Dissertação [Mestrado, Medicina Interna] Salvador: Faculdade de Medicina da Universidade Federal da Bahia, 1997;82p.

14. Gift AG. Visual analogue scales: measurement of subjective phenomena. Nurs Res 1989;38:286-288.

15. American Thoracic Society. Standardization of spirometry. 1994 update. Am J Respir Crit Care Med 1995;152:1107-1136.

16. Veen JC, Smits HH, Ravensberg AJJ, Hermelijn HS, Hiemstra HS, Sterk PJ, Bel EH. Impaired perception of dyspnea in patients with severe asthma. Am J Respir Crit Care Med 1998;158:1134-1141.

17. Kikuchi Y, Okabe S, Tamura G. Chemosensitivity and perception of dyspnea in patients with a history of near-fatal-asthma. N Engl J Med 1994;330:1329-1334.

18. Boulet LP, Leblanc P, Turcotte H. Perception scoring of induced bronchoconstriction as an index of awareness of asthma symptoms. Chest 1994;105:1430-1433.

19. Catelier P, Turcotte H, Deschesnes F, et al. Changes in lung volumes and poor perception of bronchoconstriction-induced respiratory symptoms. Ann Allergy Asthma Immunol 1998;81:315-321.

20. Roisman GL, Peiffer C, Lacronique JG, Le Cae A, Dusser DJ. Perception of bronchial obstruction in asthmatic patients. Relationship with bronchial eosinophilic inflammation and epithelial damage and effect of corticosteroid treatment. J Clin Invest 1995;96:12-21.

21. Ruffin RE, Latimer KM, Schembri DA. Longitudinal study of near fatal asthma. Chest 1991;99:77-83.

22. Salome CM, Xuan W, Gray EJ, Beloussova E, Peat JK. Perception of airway narrowing in a general population sample. Eur Respir J 1997; 10:1052-1058.

23. Laprise C, Boulet LP. Asymptomatic airway hyperresponsiveness: a three-year follow-up. Am J Respir Crit Care Med 1997;156:403-409.

24. Gavriely N. Analysis of breath sounds in bronchial provocation tests. Am J Respir Crit Care Med 1996;153:1469-1471.

25. Graviely N, Kelly KB, Grotberg JB, Loring SH. Forced expiratory wheezes are manifestation of airway flow limitation. J Appl Physiol 1987;62:2398-2403.

26. Schreur HJ, Vanderschoot J, Zwinderman AH, Dijkman JH, Sterk PJ. Abnormal lung sounds in patients with asthma during episodes with normal lung function. Chest 1994;106:91-99. 